\title{
New developments in instrumentation at the W. M. Keck Observatory
}

\author{
Sean M. Adkins ${ }^{\mathrm{e}}$, Ian S. McLean ${ }^{1 \mathrm{c}}$, Michael P. Fitzgerald ${ }^{\mathrm{c}}$, James E. Larkin ${ }^{\mathrm{c}}$, Hilton A. Lewis ${ }^{\mathrm{a}}$, \\ Christopher Martin $^{\mathrm{b}}$, Dimitri Mawet ${ }^{\mathrm{b}}$, J. X. Prochaska ${ }^{\mathrm{d}}$, Peter Wizinowich ${ }^{\mathrm{a}}$ \\ ${ }^{a}$ W. M. Keck Observatory, 65-1120 Mamalahoa Highway, Kamuela, HI, USA 96743; \\ ${ }^{\mathrm{b}}$ California Institute of Technology, 1200 E. California Blvd., Pasadena, CA 91125, \\ USA; \\ ${ }^{c}$ University of California, Los Angeles, Box 951547, Los Angeles, CA 90095-1547; \\ ${ }^{\mathrm{d}}$ University of California, Santa Cruz, 1156 High Street, Santa Cruz, CA 95064; \\ ${ }^{\mathrm{e}}$ W2 Enterprises, 77-177 Halawai Way, Kailua Kona, HI 96740
}

\begin{abstract}
The W. M. Keck Observatory is committed to maintaining the scientific leadership of our observing community by matching our observers' skills and passions in their fields of astronomical science with a continuing dedication by the Observatory and its collaborators to the development of state of the art instrumentation and systems. Our science driven strategic plan guides these developments and informs our plans for the future. In this paper we describe the performance of recently completed new instruments, instrument upgrades, and infrastructure upgrade projects. We also describe the expected performance of projects currently in the development or construction phases. Projects recently completed include a new laser for the Keck II AO system, the upgrade of the spectrograph detector in the OSIRIS instrument, and the upgrade to the telescope control system on the Keck II telescope. Projects in development include an upgrade to the telescope control system on the Keck I telescope, the blue channel of the Keck Cosmic Web Imager, the red channel of the Keck Cosmic Web Imager, the Keck Planet Finder, a deployable tertiary mirror for the Keck I telescope, an upgrade to the imager of OSIRIS, a major upgrade to the NIRSPEC instrument, and a fiber feed from the Keck II AO system to NIRSPEC.
\end{abstract}

Keywords: Adaptive Optics, Infrared, Instrumentation, Integral Field, Laser Guide Star, Spectrograph, Targets of Opportunity, Telescope Control

\section{INTRODUCTION}

Science operations at Keck I began in May 1993, and by October 1996 both telescopes were in full operation. Today, two decades later, the W. M. Keck Observatory (WMKO) has an extensive suite of powerful scientific instruments, and an impressive legacy of astronomical discoveries. Both telescopes have laser guide star adaptive optics (AO) systems, and both telescopes have a full suite of instruments. On Keck I the instruments are LRIS and MOSFIRE at the Cassegrain focus, HIRES on one Nasmyth platform, and OSIRIS with the AO system on the opposite platform. An atmospheric dispersion compensator (ADC) is available on Keck I for use with LRIS. On Keck II the Cassegrain instrument is ESI, while DEIMOS and NIRSPEC share one of the Nasmyth platforms, and NIRC2 plus the AO system occupy the other Nasmyth focus. Information about all of these instruments can been found on the WMKO web site. Of this suite of instruments, the newest is MOSFIRE, which has now been in operation for four years. WMKO's instrument suite has contributed to numerous scientific discoveries ranging from solar system bodies and exoplanets, to the galactic center, to supernovae, and the high redshift universe. Developing new instrumentation, improving facilities, and upgrading popular instruments, continues to be an important part of our science driven strategic plan. In recent years we have been engaged in upgrades to LRIS and OSIRIS, and to the AO systems. The next new instruments will be KCWI and NIRES. Work is also under way on a deployable tertiary mirror for Keck 1. Currently, the Observatory is engaged in a new round of strategic planning which is being developed by the Science Steering Committee (SSC) in partnership with Observatory staff and the astronomical

${ }^{1}$ mclean@astro.ucla.edu; Phone: (310) 825-1140; https://irlab.astro.ucla.edu/

Ground-based and Airborne Instrumentation for Astronomy VI, edited by Christopher J. Evans, Luc Simard, Hideki Takami Proc. of SPIE Vol. 9908, 990805 - (c) 2016 SPIE · CCC code: 0277-786X/16/\$18 · doi: 10.1117/12.2233759 
community. Developments up through 2014 were described at the last SPIE conference in this series. In this paper we describe progress on new instrumentation and upgrades since our last overview [1].

\section{NEW DEVELOPMENTS IN INSTRUMENTATION}

\subsection{New laser for the Keck II AO system}

A new laser has replaced the dye laser on the Keck II telescope's laser guide star (LGS) adaptive optics (AO) system [2]. Referred to as the next generation laser (NGL), the laser was developed by TOPTICA Photonics AG and MPB Communications Inc. in collaboration with the European Southern Observatory and the Thirty Meter Telescope project. The new laser generates $19 \mathrm{~W}$ of optical power at the sodium D2a line, and $1.9 \mathrm{~W}$ of power at the sodium D2b line. This two-line operation allows "re-pumping" of the sodium atoms from the lower ground state into higher states allowing renewed photon interaction and significantly increasing the brightness of the LGS image. On sky testing of the laser began in December 2015 with a center launch system (CLS). Figure 1 shows recent results from the laser's testing, demonstrating better beam quality and a 2.7 magnitude improvement in the sodium return as compared to the initial dye laser using a side launch system (SLS). The new laser's return also shows the expected dependence on azimuth pointing due to the earth's magnetic field as demonstrated by Figure 2 which shows the return in $\mathrm{R}$ magnitude for different pointing directions. The laser is now in use for science observing.

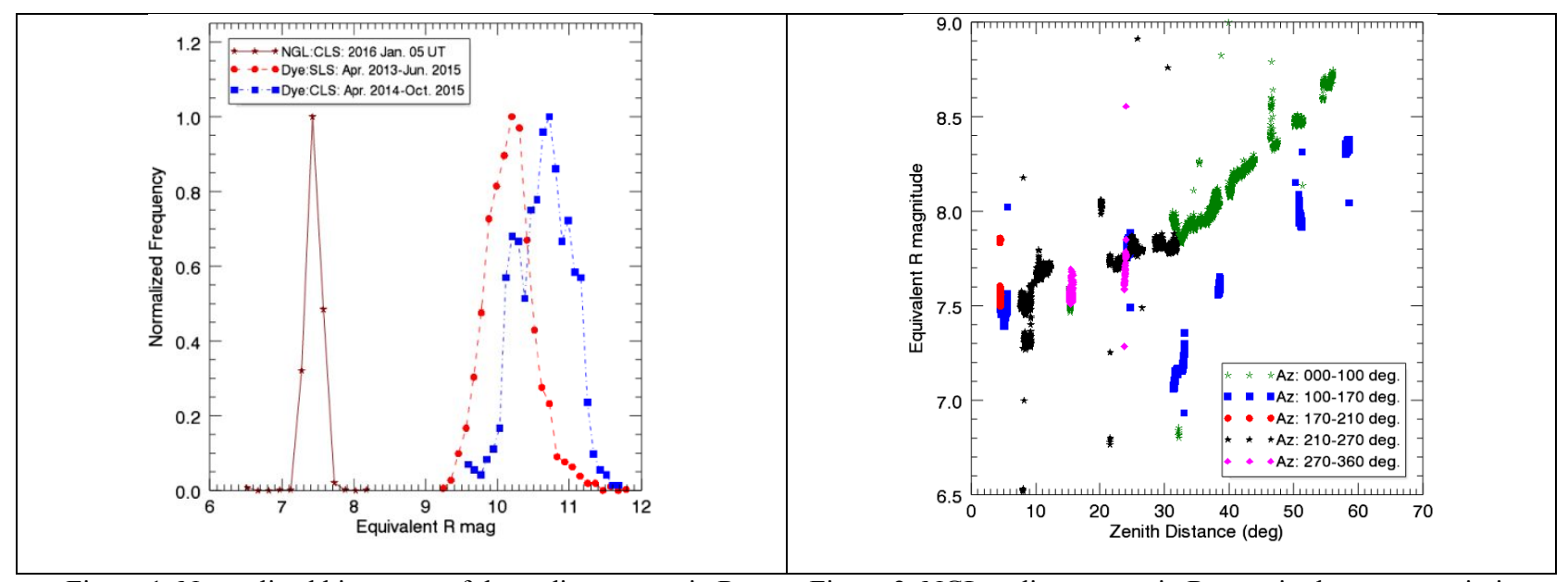

Figure 1: Normalized histogram of the sodium return in $\mathrm{R}$ magnitudes of the NGL with CLS, dye Laser with CLS, and

Figure 2: NGL sodium return in R magnitudes versus pointing the dye laser with SLS

\subsection{Upgrade of the spectrograph detector in the OSIRIS instrument}

The OSIRIS integral field spectrograph (IFS) [3] used with the Keck I AO system has received an upgrade to its spectrograph detector [5]. OSIRIS is a near-IR IFS covering the wavelength range of 1 to $2.4 \mu \mathrm{m}$, with spatial sampling scales of 20, 35, 50, and 100 milliarcseconds. With support from the National Science Foundation (NSF) the University of California, Los Angeles (UCLA) Infrared Laboratory has collaborated with WMKO to design and implement a new detector head for OSIRIS, replacing the instrument's Hawaii-II detector and ARC generation III readout system with a Hawaii-2RG and a Sidecar ASIC based readout system. The new detector head also incorporated a focus stage to assist in verifying alignment by allowing through focus motion to measure tip-tilt. After two cool downs the alignment and focusing of the detector was completed. The upgrade has delivered a significant improvement to the instrument's sensitivity as shown in Table 1. The detector upgrade compliments the grating upgrade completed in 2012 [4] and together the two upgrades have increased the sensitivity of the instrument by 3 to 4 times. The instrument returned to science operations on April 18, 2016. 


\begin{tabular}{|l|l|l|l|l|}
\hline Band & Filter & $\begin{array}{l}\text { Central } \\
\text { Wavelength }(\boldsymbol{\mu m})\end{array}$ & $\begin{array}{l}\text { Sampling Scale } \\
\text { (milliarcseconds) }\end{array}$ & $\begin{array}{l}\text { Throughput Gain } \\
\text { New/old Detector }\end{array}$ \\
\hline J & Jn1 & 1.20 & 100 & 2.04 \\
\hline J & Jn2 & 1.26 & 35 & 2.09 \\
\hline H & Hn2 & 1.57 & 100 & 1.68 \\
\hline K & Kn3 & 2.17 & 35 & 1.63 \\
\hline
\end{tabular}

Table 1: Throughput gain from the new spectrograph detector in $\mathrm{J}, \mathrm{H}$, and $\mathrm{K}$ bands. Gain is the ratio of raw frame flux in electrons for the same star observed using the old Hawaii-2 detector and the new Hawaii-2RG detector in the same filter and sampling scale.

\subsection{Upgrade to the telescope control system on the Keck II telescope}

The installation of the upgrade to the telescope control system of the Keck II telescope was completed in November 2015. On sky testing has demonstrated a significant improvement in blind pointing accuracy as well as improvements in slew speed and open loop tracking performance [6]. The system passed its operational readiness review in March 2016 and was first used for science on June 16, 2016. The system is being released for use with each of the Keck II instruments on a progressive basis, the system will be released for all Keck II instruments by the end of July 2016.

On-sky blind pointing performance is 1.24" rms using a model constructed with 7 months' pointing test data. The best model fit ranges from 0.68 " to 0.87 " rms. This may be compared to the original system where the model fit was $\sim 5^{\prime \prime}$ and frequently showed much worse on-sky blind pointing performance. A final tuning of the servo was completed in May 2016 with the assistance of servo control expert Peter Thompson [7]. The system is now meeting tracking requirements and shows improved wind-shake rejection. A conservative estimate of the time saved by the improved pointing performance is 5 nights/year, determined using 1 years' worth of actual acquisitions.

\subsection{Upgrade to the telescope control system on the Keck I telescope}

The installation of the telescope control system upgrade on the Keck I telescope is complete and testing is in progress. The first night-time engineering tests were conducted in May 2016. The best model fit is $<1.4 "$ rms. The Keck I upgrade was successfully pressed into service for one MOSFIRE science night due to a fault with the existing system. It is anticipated that the remaining Keck I system engineering tests and on-sky tests with each Keck I science instrument will be completed by the end of September 2016.

\subsection{Blue channel of the Keck Cosmic Web Imager}

KCWI, the Keck Cosmic Web Imager, is an integral field spectrograph for the visible wavelengths being developed for the Keck II telescope by the California Institute of Technology, UCLA, the University of California, Santa Cruz, (UCSC), and WMKO. KCWI will provide seeing-limited integral field spectroscopy with moderate to high spectral resolution, high efficiency, and excellent sky subtraction with available nod and shuffle capability. KCWI is a two channel instrument designed for phased implementation with the blue channel covering the wavelength range of 350 to $560 \mathrm{~nm}$, and the red channel covering 530 to $1050 \mathrm{~nm}$. The instrument's key performance parameters are shown in Table 2.

\begin{tabular}{|c|c|}
\hline Parameter & Value \\
\hline Field of View & Selectable: $20 " \mathrm{x}(8.4|16.8| 33.6) "$ \\
\hline Spatial Res./Sampling & Selectable: $0.35 " \mathrm{x}(0.35|0.7| 1.4) "$ \\
\hline Spectral Resolution & Selectable: 1,000 to 20,000 \\
\hline Bandpass (red \& blue) & 350 to $1050 \mathrm{~nm}$ \\
\hline Efficiency & $>40 \%$ (instrument) \\
\hline $3 \sigma$ Sensitivity in 1 Hour & $10^{-7}$ to $10^{-6} \mathrm{ph} / \mathrm{s} / \mathrm{cm}^{2} / \operatorname{arcseccond}^{2} / \AA$ \\
\hline Light Bucket Sensitivity & 200 LU in 10 hours \\
\hline Background Subtraction & $0.01 \%$ of sky \\
\hline Plate Scale & $0.15^{\prime \prime}$ pixel $^{-1}$ \\
\hline
\end{tabular}

Table 2: KCWI key performance parameters 
The instrument with the blue channel is currently in the full scale development phase. The blue channel's passband covers 350 to $560 \mathrm{~nm}$. A selection of 5 gratings is provided for the blue channel allowing spectral resolutions from 1,000 to 20,000 . The instrument's opto-mechanical assembly [8] and alignment is complete. The work remaining to complete the instrument consists of the fabrication and testing of the cart and jacking system needed to move the instrument from a storage position to the in-beam position at the Keck II right Nasmyth focus, and the fabrication and installation of the instrument's light tight enclosure. Delivery of the completed instrument is expected in the late fall of 2016 with first light in early 2017.

\subsection{Red channel of the Keck Cosmic Web Imager}

The completion of the blue channel instrument in 2016 will be followed by the design and construction of a red channel upgrade for KCWI. This will provide a passband from 530 to $1050 \mathrm{~nm}$ with a deep depletion red optimized $\mathrm{CCD}$ and a selection of 7 gratings and 6 selectable order sorting filters. The red channel will share the k-mirror image de-rotator, calibration unit, IFU, and collimator optics with the blue channel via a dichroic beam splitter. The red upgrade will also require the addition of an off-axis tracking guider. The blue channel only version of the instrument shares the science field $\mathrm{k}$-mirror with a guide camera via small dichroic beam splitter that sends the red channel light to an on-axis camera. This was done for cost reasons, and will be removed when the red channel upgrade is installed. The current project plan schedules delivery of the upgrade to the observatory in late 2018. After installation on the KCWI instrument first light for the red channel upgrade is expected in March 2019.

\subsection{The Keck Planet Finder}

The Keck Planet Finder [9] is a fiber fed visible wavelength (400 $\mathrm{nm}$ to $870 \mathrm{~nm}$ with two channels) spectrograph optimized for precision radial velocity observations. The instrument is being developed in collaboration with the University of California, Berkeley's Space Sciences Laboratory and the University of Hawaii. A block diagram of the instrument is shown in Figure 3.

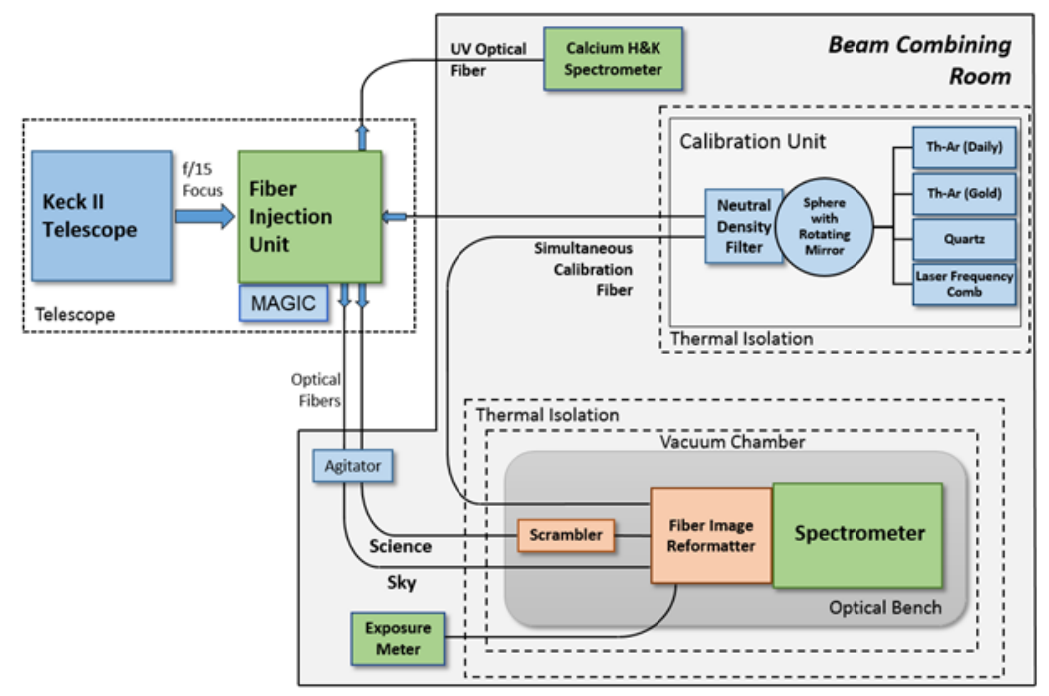

Figure 3: Block Diagram of the Keck Planet Finder

Light from the Keck II telescope will feed the Keck Planet Finder Fiber Injection Unit (FIU), which will be located on the Nasmyth platform of the Keck II telescope. This system will include an atmospheric dispersion corrector (ADC), a tip/tilt mirror for image stabilization, a calibration light input, and the acquisition and guide camera. The FIU also focuses light from the telescope onto a science fiber, a sky fiber (to monitor contamination from moonlight and the sky background), and a fiber sending UV light to a small spectrometer monitoring the Calcium $\mathrm{H} \& \mathrm{~K}$ spectral lines (to monitor contamination from stellar activity).

The science and sky fibers will travel $70 \mathrm{~m}$ to the Keck Planet Finder spectrometer, located in the beam combining room in the observatory basement. In order to isolate the spectrometer from its environment, the spectrometer will be placed within a vacuum chamber (for isolation against changes in atmospheric pressure), and within a pair of 
nested, insulated rooms (for isolation against temperature changes at the milli-Kelvin level, which can affect the spectrometer through expansion and contraction).

The optical fiber to feed allows the needed isolation between the spectrograph and the motion of the telescope and also provides an effective "scrambler" that increases the illumination stability at the spectrograph entrance. To improve the scrambling performance, the science fiber will be an octagonal fiber, and the fiber system will include a scrambler system, consisting either of a small ball lens, or a pair of lenses, that swap the near and far fields of the fiber output. This system then feeds a short length of octagonal fiber to further homogenize the scrambler output. Fiber modal noise can also cause illumination instabilities as the output of a fiber, and to mitigate that effect an agitator will be employed to mechanically move the science and sky fibers. Following the scrambler, the science fiber system ends at the input to the reformatter. This system takes the light leaving the fiber, and rearranges it into three "slices", aligned along the spectrometer (virtual) slit. This system effectively allows the Keck Planet Finder spectrometer to be three times smaller than a traditional spectrometer would be, given the aperture and image scale of the Keck telescopes.

The sky fiber also terminates at the reformatter. Although the sky fiber is not reformatted like the science fiber, its light passes through the reformatter and is converted to the $\mathrm{f} / 8$ focal ratio that the core spectrometer accepts.

Also shown in Figure 3 is a calibration system, which will reside in the observatory basement near the spectrometer. This will consist of the typical spectrometer calibration sources (Thorium-Argon lamps for wavelength calibration and a quartz lamp for flat fielding). There will also be provision for future, high line density calibration sources like Fabry-Perot etalons or laser frequency combs to be easily added to the system. These sources will feed an integrating sphere (with a rotating mirror inside to mitigate modal noise effects), which will then feed two fibers. One fiber will travel up to the telescope, where the FIU will focus the calibration light it contains onto the science and sky fibers. The second fiber will travel directly to the spectrometer, pass through the reformatter as the sky fiber does (i.e. without reformatting), and provide a simultaneous calibration source during a science exposure.

Also included within the KPF system is a separate, small spectrometer to monitor the Calcium H \& K spectral lines (for stellar activity that could mimic Doppler shifts), and an exposure meter (to determine the flux-weighted midpoint of each spectrum, for accurate barycentric corrections).

The Keck Planet Finder has completed its system design phase, and is now in the preliminary design phase with funding from the Heising-Simons Foundation. The preliminary design review is planned for 2017, and first light is expected in 2019.

\subsection{A deployable tertiary mirror for the Keck I telescope}

With funding from an NSF MRI grant, the University of California Observatories (UCO) on the UCSC campus is collaborating with WMKO in the development of a deployable tertiary mirror for the Keck I telescope, called the "Keck 1 Deployable Tertiary Mirror" or "K1DM3" [10]. The K1DM3 is a module based on the design of the existing tertiary mirror module, but employing a smaller mirror to make retraction from the beam practical. While the Keck telescopes support a 20' of view (FOV), the current Nasmyth platform instruments on Keck I (HIRES and Keck I AO with OSIRIS) require much smaller FOVs. The K1DM3 supports an un-vignetted FOV of 4.7'. The K1DM3 module will remain in the telescope at all times and can move between the deployed and retracted positions in less than $120 \mathrm{~s}$. This will allow observers to select between the currently installed Cassegrain instrument (LRIS or MOSFIRE), and the two Nasmyth instruments, HIRES or OSIRIS.

Figure 4 shows the K1MD3 in the retracted and deployed configurations. The K1DM3 has completed its detailed design review and is in the full scale development phase. Delivery to the observatory is expected in late 2016 with completion of commissioning planned for mid-2017. 

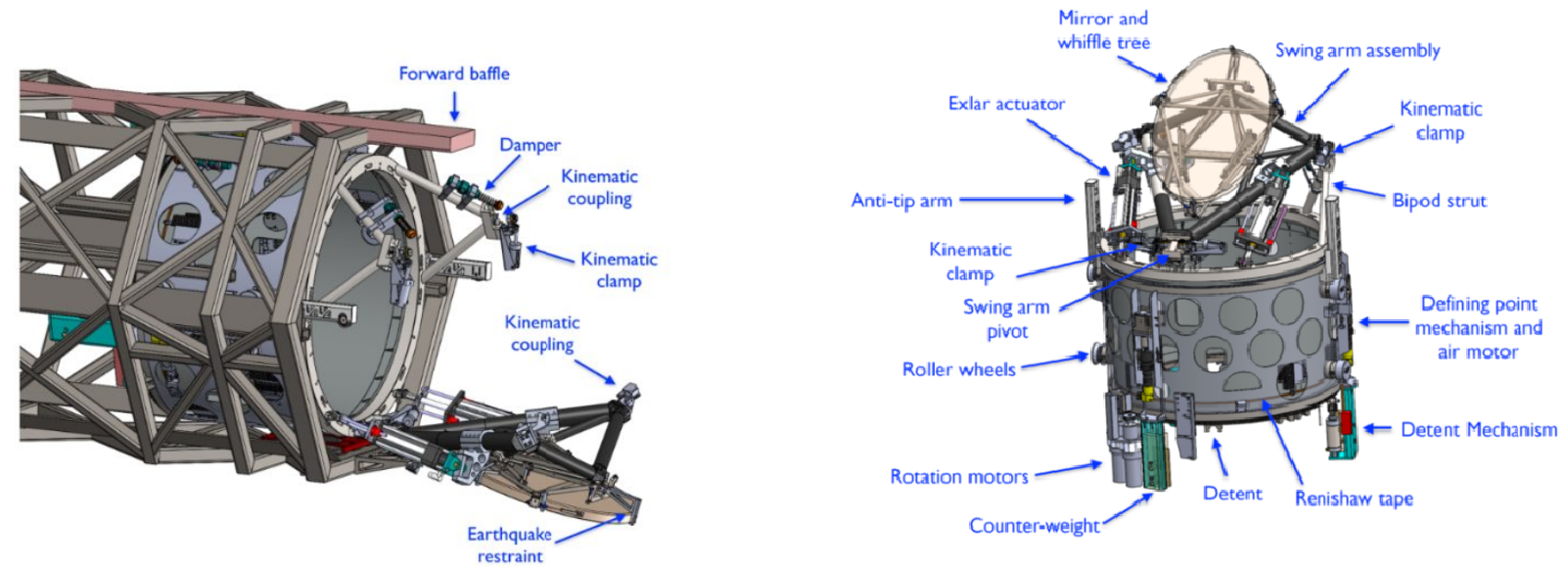

Figure 4: K1DM3 in retracted (left) and deployed (right) configurations

\subsection{An upgrade to the imager of OSIRIS}

UCLA and the observatory are also collaborating on the development of an upgrade to the imager for the OSIRIS instrument [11]. The imager upgrade will replace the entire imager optical path and uses a Hawaii-2RG detector to provide $\sim 9$ milliarsecond/pixel resolution with an 18.4" x 18.4" FOV. This FOV is large enough to image all of the well known astrometric references used to establish the astrometric frame used for synoptic observations of the Galactic Center. The upgrade is designed to provide low distortion $(\sim 1 \%)$ and will also provide increased sensitivity due to the higher quantum efficiency $(\mathrm{QE})$ of the Hawaii-2RG. The optical design provides a well formed pupil allowing a high efficiency cold stop. The upgrade also increases the number of filters available by providing two new filter wheels with 18 positions each. One of the wheels is located at the pupil plane with some positions being used for selectable size cold stops. The opto-mechanical layout of the upgrade is shown in Figure 5.

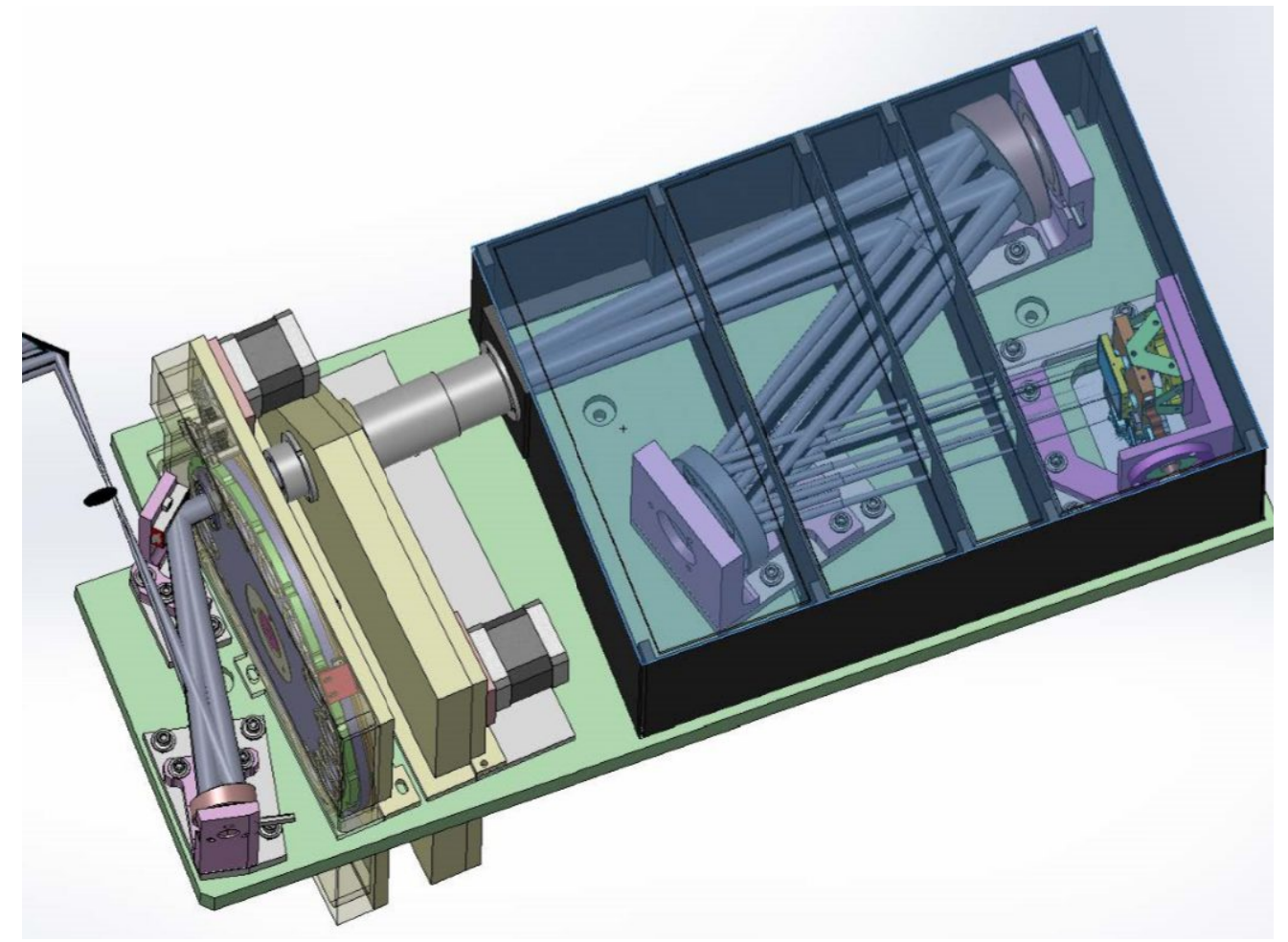

Figure 5: OSIRIS Imager Upgrade Opto-mechanical Layout 
The upgrade completed a design review in April 2016 and installation is planned for late in 2016 with commissioning in early 2017.

\subsection{A major upgrade to the NIRSPEC instrument}

Near-IR spectroscopy from 1 to $5 \mu \mathrm{m}$ with NIRSPEC, the Near-IR SPECtrometer [12][13] on the Keck II telescope at WMKO has been responsible for numerous important discoveries since its first on-sky observations in 1999. Since that time considerable progress has been made in infrared detector technology, and many of NIRESPEC's supporting systems, especially those used for detector readout and control are obsolete. The NIRSPEC upgrade [14] will replace the spectrograph's 1024 x 1024 pixel Aladdin III detector with a $5 \mu$ m cutoff Hawaii-2RG. The smaller pixels will increase spectral resolution from 25,000 (0.43" slit, 3-pixel sampling to 37,500 (0.29" slit, 4 -pixel sampling). The lower noise and greater $\mathrm{QE}$ of the Hawaii-2RG will improve sensitivity by 1 magnitude for wavelengths shorter than $2 \mu \mathrm{m}$. Figure 6 shows the predicted gain in SNR at various wavelengths with the Hawaii2RG. Compared to the Aladdin 3 the Hawaii-2RG offers at least a 5-fold reduction in read noise (4.5 e- for the Hawaii-2RG vs. 23 e- with 16 Fowler samples), and as much as a 70 -fold reduction in dark current (as low as 0.01 e-/s for the Hawaii-2RG vs. 0.7 e-/s).

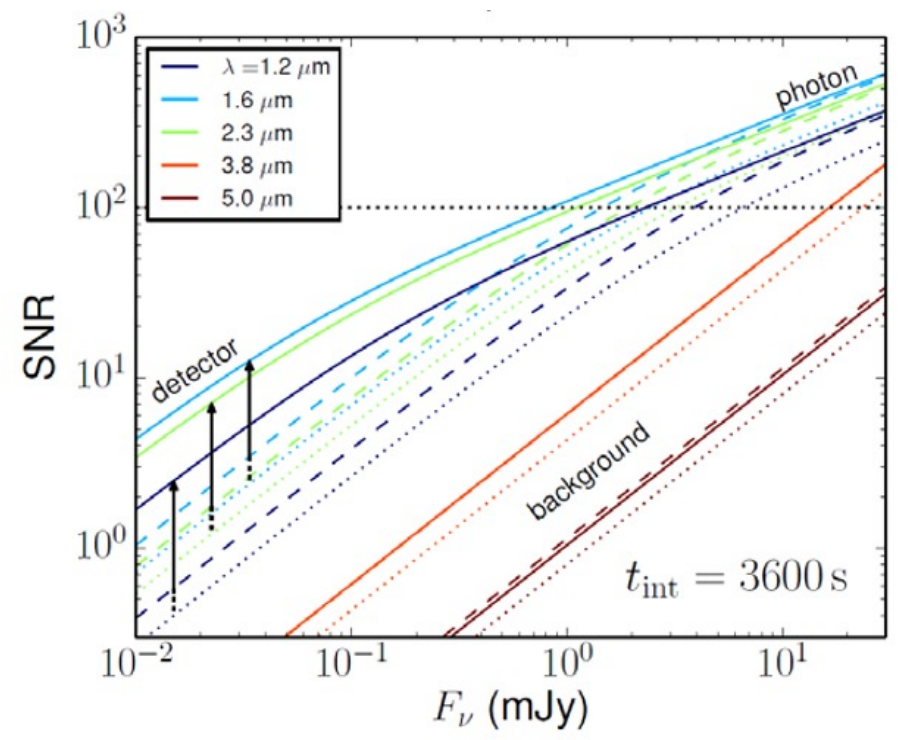

Figure 6: Spectrograph SNR improvement vs. flux level for a 1-hour exposure at representative wavelengths in each IR band.

The dashed lines show the current performance with the Aladdin 3, and the dotted lines show the loss in SNR due to the intermittent excess noise in the current system. The solid lines show the predicted improvement with the Hawaii-2RG (indicated by the vertical arrows). The gain is most dramatic for faint sources.

The upgrade will also replace the slit viewing camera optics and detector extending the passband of the slit viewing camera to $5 \mu \mathrm{m}$ [15]. This upgrade will use a Hawaii-1RG detector, and both the slit viewing camera and spectrograph detector upgrades will use the Sidecar ASIC and Sidecar Acquisition Module (also used in the OSIRIS upgrades). The slit viewing camera is fed with light reflected from the spectrograph slits, and while in-band guiding for $\mathrm{L}$ and $\mathrm{M}$ band will often be an improvement, especially for faint sources, provisions will be made to allow a mode where the thermal IR ( $>2 \mu \mathrm{m})$ is cut-off allowing out of band guiding on bright sources

We will also investigate how the spectral line calibration of the spectrograph can be improved with the goal of improving the precision of radial velocity measurements. Finally, new mechanism control and temperature monitoring and control systems will share a common spares pool with MOSFIRE and increase NIRSPEC's useful lifetime and maintainability. New server and control computers will accompany a complete upgrade of the instrument's software with a modern client-server architecture based on the proven software developed for MOSFIRE. Instrument user interface (UI) software has also progressed with better graphical UI development environments and the NIRSPEC upgrade will take advantage of the UI improvements introduced with MOSFIRE. This commonality of software and user interface design will reduce the time needed to help new observers gain 
familiarity with NIRSPEC and will also improve the response time for software support because of the shared code base with MOSFIRE and future WMKO instruments such as KCWI.

The upgrade is currently in the design phase with a design review planned for later this year. Installation of the upgrade will start approximately one year later, with first light in early 2018.

\subsection{A fiber feed from the Keck II AO system to NIRSPEC}

A fiber optic feed from the Keck II AO system to NIRSPEC is under development with funding from the HeisingSimons Foundation. Initially planned as a K-band feed [16] this will be expanded to allow an H-band feed as well, along with precision radially velocity calibration using a laser frequency comb. The K-band fiber feed will be combined with a vortex coronagraph mask in the AO system for Doppler imaging.

\section{CONCLUSIONS}

WMKO has continued to develop new capabilities to serve its world leading community of observers. A new strategic plan is in development that will guide instrumentation efforts in the future, this plan is being developed with substantial participation from the national community and from our growing international partnership as well. As we develop new instruments we also look for opportunities to upgrade our existing ones. Such upgrades have proven to be very rewarding in terms of science gain and improved operational stability and maintainability. We expect to maintain this combined approach as we continue the Observatory's third decade.

\section{ACKNOWLEDGEMENTS}

The W. M. Keck Observatory is operated as a scientific partnership among the California Institute of Technology, the University of California, and the National Aeronautics and Space Administration. The Observatory was made possible by the generous financial support of the W. M. Keck Foundation.

The development of the Keck II next generation laser is made possible through the support of the Gordon and Betty Moore Foundation, the W. M. Keck Foundation, the Bob \& Renee Parsons Foundation, Friends of Keck Observatory, and the Thirty Meter Telescope Project.

The development of the blue channel of KCWI is supported in part by a grant from National Science Foundation under Scientific Program Order No. 5 as issued for support of the Telescope Systems Instrumentation Program (TSIP), in accordance with Proposal No. AST-0335461 submitted by AURA. Support for KCWI has also come from the Heising-Simons Foundation and the Mount Cuba Foundation.

Observatory operations are supported in part by the National Aeronautics and Space Administration under Grant and Cooperative Agreement No. NNX13AH26A. The OSIRIS spectrograph upgrade is supported by the National Science Foundation under Grant No. AST-1311102, K1DM3 is supported in part by the National Science Foundation under Grant No. AST-1337609, the red channel of KCWI is supported in part by the National Science Foundation under Grant No. AST-1429890. The NIRSPEC upgrade is supported in part by the National Science Foundation under Grant No. AST-1532315, and by the Heising-Simons Foundation. The OSIRIS grating upgrade project was funded in part by the Dunlap Institute, University of Toronto. The OSIRIS imager upgrade project is supported by funding from the Gordon and Betty Moore Foundation. Development of KPF is supported by the Heising-Simons Foundation.

\section{REFERENCES}

[1] Adkins, Sean M., Armandroff, Taft E., Fitzgerald, Michael P., Johnson, James, Larkin, James E., Lewis, Hilton A., Martin, Christopher, Matthews, Keith Y., Prochaska, J. X., and Wizinowich, Peter, "New developments in instrumentation at the W. M. Keck Observatory", Proc. SPIE 9147, 914703 (2014).

[2] Chin, Jason C., Wizinowich, Peter L., Wetherell, Edward, Lilley, Scott J., Cetre, Sylvain, Ragland, Sam, Medeiros, Drew W., Tsubota, Kevin, Doppmann, Greg, Otárola, Angel C., and Wei, Kai, "Keck II laser guide star AO system and performance with the TOPTICA/MPBC laser", Proc. SPIE 9909, 29 (2016). 
[3] Larkin, James, Barczys, Matthew, Krabbe, Alfred, Adkins, Sean, Aliado, Ted, Amico, Paola, Brims, George, Campbell, Randy, Canfield, John, Gasaway, Thomas, Honey, Allan, Iserlohe, Christof, Johnson, Chris, Kress, Evan, LaFreniere, David, Magnone, Ken, Magnone, Nick, McElwain, Michael, Moon, Juleen, Quirrenbach, Andreas, Skulason, Gunnar, Song, Inseok, Spencer, Michael, Weiss, Jason, and Wright, Shelley, "OSIRIS: A diffraction limited integral field spectrograph for Keck", New Astronomy Reviews 50(4-5), 362-364 (2006).

[4] Mieda, Etsuko, Wright, Shelley A., Larkin, James E., Graham, James R., Adkins, Sean M., Lyke, James E., Campbell, Randy D., Maire, Jérôme, Do, Tuan, and Gordon, Jacob, "Efficiency Measurements and Installation of a New Grating for the OSIRIS Spectrograph at Keck Observatory", Publications of the Astronomical Society of Pacific 126(937), 250-263 (2014).

[5] Boehle, Anna, Larkin, James E., Adkins, Sean M. Aliado, Theodore, Fitzgerald, Michael P., Johnson, Christopher A., Lyke, James E., Magnone, Kenneth G., Sohn, Ji Man, Wang, Eric, and Weiss, Jason L., "Upgrade of the detector in the integral field spectrograph OSIRIS at the W. M. Keck Observatory", Proc. SPIE 9908, 104 (2016).

[6] Kwok, Shui Hung, Krasuski, Tomas, Lyke, James E., Mader, Jeffery A., McCann, Kevin L., and Tsubota, Kevin, "Improving the pointing and tracking performance of the Keck telescopes", Proc. SPIE 9913, 106 (2016).

[7] Thompson, Peter M., Krasuski, Tomas, Tsubota, Kevin, Kwok, Shui Hung, and Fumo, John, "A new two path shaper and feedforward control architecture for the Keck telescopes", Proc. SPIE 9906, 3 (2016).

[8] Rockosi, Constance, Cowley, David J., Cabak, Gerald, Hilyard, David, and Pfister, Terry, "Blue camera of the Keck cosmic web imager, fabrication and testing”, Proc. SPIE 9908, 192 (2016).

[9] Gibson, Steven R., Howard, Andrew W., Marcy, Geoffrey W., Edelstein, Jerry, Wishnow, Edward H., and Poppett, Claire L., "KPF: Keck Planet Finder", Proc. SPIE 9908, 265 (2016).

[10] Prochaska, Jason X., Ratliff, Christopher T., Cabak, Gerald, Tripsas, Alex, Adkins, Sean, Bolte, Michael, Cowley, David, Dahler, Mike, Deich, William, Lewis, Hilton, Nelson, Jerry, Park, Sam, Peck, Michael, Phillips, Andrew, Pollard, Mike, Randolph, Bill, Sandford, Dale, Ward, James, and Wold, Truman, "Detailed design of a deployable tertiary mirror for the Keck I telescope", Proc. SPIE 9910, 97 (2016).

[11]Fitzgerald, Michael P., Aliado, Theodore, Arriaga, Pauline, Boehle, Anna, Canfield, John M., Do, Tuan, Esposito, Thomas, Ghez, Andrea M., Johnson, Christopher A., Larkin, James E., Magnone, Kenneth G., Wang, Eric, Weiss, Jason L., Witzel, Gunther, Yelda, Sylvana, Kupke, Renate, Adkins, Sean M., Chock, Jon, Honey, Allan, Lyke, James E., and Wizinowich, Peter L., "Motivation for and design of a new imaging camera for the OSIRIS instrument at Keck", Proc. SPIE 9908, 92 (2016).

[12] McLean, I. S., Becklin, E. E., Bendiksen, O., Brims, G., Canfield, J., Figer, D. F., Graham, J. R., Hare, J., Lacayanga, F., Larkin, J. E., Larson, S. B., Levenson, N., Magnone, N., Teplitz, H., and Wong, W. "Design and development of NIRSPEC: a near-infrared echelle spectrograph for the Keck II telescope", Proc. SPIE 3354, 566-578 (1998).

[13] McLean, I. S., Becklin, E. E., Figer, D. F., Larson, S., and Graham, J., "NIRSPEC: a near-infrared crossdispersed echelle spectrograph for the Keck II telescope", Proc. SPIE 2475, 350-358 (1995).

[14] Martin, E. C., Fitzgerald, M. P., McLean, I. S., Adkins, S. M., Aliado, T., Brims, G., Johnson, C., Magnone, K., Wang., E., and Weiss, J., "Performance Modeling of an Upgraded NIRSPEC on Keck", Proc. SPIE 9147, 914781 (2014).

[15] Martin, Emily C., Fitzgerald, Michael P., McLean, Ian S., and Wang, Eric, "Optical design of the slit-viewing camera for the NIRSPEC upgrade", Proc. SPIE 9908, 105 (2016).

[16] Mawet, Dimitri, "Keck planet imager and characterizer (KPIC): concept and phased implementation", Proc. SPIE 9909, 13 (2016). 